\title{
Visual Stimulation as Pain Relief for Hong Kong Chinese Patients with Leg Ulcers
}

\author{
MIMI M.Y. TSE, Ph.D., ${ }^{1}$ JACOBUS K.F. NG, M.B.Ch.B., FANZA, FHKCA, FHRM (Anaes), ${ }^{2}$ and \\ JOANNE W.Y. CHUNG, Ph.D. ${ }^{1}$
}

\begin{abstract}
Analgesic potential of visual stimulation was examined in 33 patients with leg ulcers in a randomized, controlled, crossover clinical trial. Patients were alternating between wearing an eyeglass display with soundless VCD broadcast (V-sessions) and a static blank screen (B-sessions) while receiving superficial debridement and wound dressing for their leg ulcers. A significant reduction in pain scores was found during V-sessions (VAS $67.7 \pm 28.4$ vs. $25.6 \pm$ $\mathbf{2 9 . 8}$ when V-sessions vs. B-sessions, with $p<0.01$ ). Age was positively correlated with the improvement in VAS, whereas gender, residency, and the underlying medical conditions were not correlated with the improvement in pain score. The use of visual stimulation might be beneficial to both genders, in an older age group regardless of the underlying medical conditions. This is the pioneer use of visual stimulation as a non-pharmacological adjuvant to pain relief among a local Chinese population. The study will certainly add knowledge to the existing pain relief methods.
\end{abstract}

\section{INTRODUCTION}

$\mathrm{C}^{\prime}$ HRONIC ULCERATION OF THE LEG is a condition more prevalent in the elderly population. ${ }^{1-3}$ Arterial venous insufficiencies, as well as microvascular problems such as that associated with diabetes are the common causes. ${ }^{4}$ Chronic leg ulcers are slow in healing and have a high likelihood of recurrence. ${ }^{5}$ Patients may become very frustrated. ${ }^{6}$

A previous study evaluating chronic leg ulcers ${ }^{2}$ showed that patients tend to have high pain scores, emotional reactions, social isolation and impaired physical mobility. In another study, pain was found to be "the worst thing about having a leg ulcer."7 Pain was continuous, difficult to control and had a negative impact on the daily life of the elderly.

It was alarming to find in one study that $38 \%$ of patients were receiving no analgesia at all despite the fact that they had expressed pain in relation to their leg ulcers. ${ }^{2}$ Patients tend to avoid situations such as standing, walking and going out that might trigger more pain. ${ }^{7}$ As a result, the elderly patients are more likely to become inactive and socially isolated.

The debridement of leg ulcers is necessary to remove sloughs, fibrinous tissue and necrosis to promote granulation and healing. ${ }^{8}$ Yet, it is often very painful, and inadequate pain relief may hinder the satisfactory cleansing of many leg ulcers. ${ }^{9}$ It has been suggested that leg ulcers may require strong pain relief prior to dressing changes. ${ }^{10}$

Melzack and Wall ${ }^{11}$ suggest that pain experiences consist of three dimensions: (1) sensorydiscriminative, (2) motivational-affective, and (3) cognitive control processes. The transmission of nerve impulses to spinal cord transmission (T) cells is modulated by a spinal gating mechanism in the dorsal horn. According to Melzack,12 selective cognitive processes are activated by a specialized system of large-diameter fibers and have the property of modulating the spinal gating mechanism by the descending fibers. In this situation, cognitive activities such as distraction can close 
the gate and prevent or modulate the sensation of pain. Indeed, Sparks ${ }^{13}$ proposes the use of cognitive distraction to alter pain perception, using the conceptual framework of the gate control theory. The effects of two forms of distraction, touching and bubble blowing, were compared in 105 preschool children for injection pain. The findings of this study ${ }^{13}$ indicated that both forms of distraction resulted in significantly reduced pain perception.

Distraction is one of the important uses of cognitive-behavioral techniques to relieve pain as suggested by the Gate Control theory. Music therapy is an effective sensory technique for distraction. ${ }^{14}$ The therapeutic use of music therapy has been well documented experimentally and clinically as an effective adjunct for anxiety and pain, resulting in an improvement in patient comfort. ${ }^{15-18}$ The use of visual stimulation might be as effective as auditory stimulation for pain relief. A kaleidoscope has been used to generate visual stimulation in a routine blood draw with promising results. ${ }^{19}$

To this end, the application of visual stimulation via an eyeglass display as an adjunct to pain relief during leg ulcer dressing in the community patients was studied. In this study, the effect of gender, age, residency, underlying medical conditions, the degree of enjoyment and memory of the content in the VCD on any observed analgesic effect was also explored.

\section{MATERIALS AND METHODS}

It was a randomized, controlled, cross-over clinical trial. Patients were randomly allocated to two sessions with subsequent crossover. After approval by the Human Ethics Committee of the University of Hong Kong and the Hospital Chief Executive of Kowloon Hospital, a convenience sample of 33 patients was recruited for this study. Inclusion criteria were adult patients with a normal cognitive function (rate 8-10) as evaluated by the Abbreviated Mental Test-Modified (AMT) who need superficial debridement and dressing for leg ulcers. Patients with cancer were excluded from this study. All subjects were informed of the purpose and procedure of the study, and were free to withdraw from the study at any time. Informed consent was obtained from all subjects.

In this study, an Olympic Eyetreck eyeglass was used and was connected to a VCD (Fig. 1). The eyeglass only weighs $120 \mathrm{~g}$ and could easily slip onto the user's face as a pair of spectacles (Fig. 2). Wear-

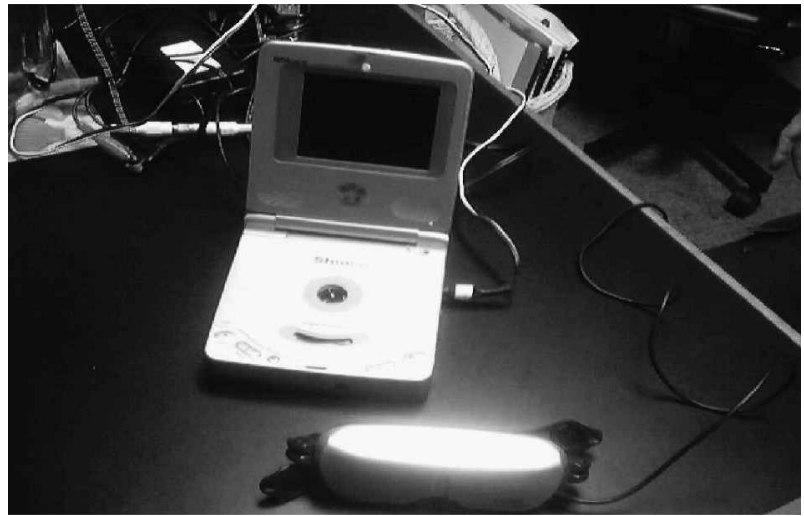

FIG. 1. The Olympic Eyetreck eyeglass was connected to a VCD.

ing the eyeglass display gives the feeling of watching a 52 -inch television screen from only $6 \frac{1}{2}$ feet away.

Patients were randomly assigned to participate in either a V session or a B session first while having their wound dressing and debridement for the leg ulcers. In $\mathrm{V}$ session, patients were instructed to wear a soundless video display eyeglass, of which the visual content was selected according to the preferences of the patients (ranging from Chinese opera to cartoons and natural environment of mountains and waterfall). In B session, patients watched a static blank screen via the eyeglass. The study was conducted over 2 separate days. Patients allocated to $\mathrm{V}$ session on the first day would be allocated to $B$ session on the second day and vice versa. As a usual practice, no other analgesics were administered during these sessions.

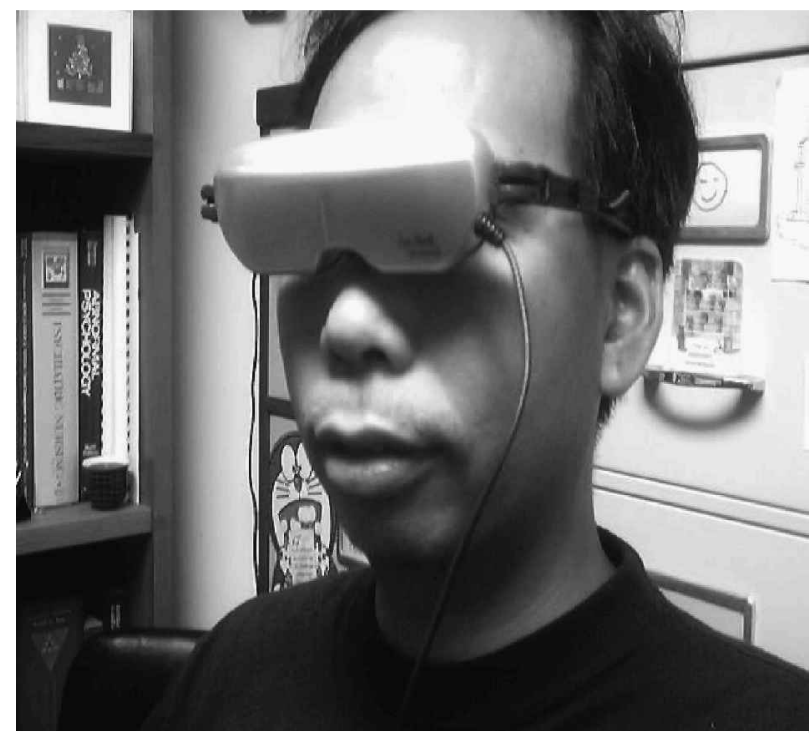

FIG. 2. Wearing the eyeglass display. 
Pain intensity was assessed immediately after the procedure using the Visual Analogue Scale (VAS). Also, patients were asked the degree of enjoyment in watching the videotape using numerical anchors ranging from 0 to 10 , where $0=$ not enjoy at all and $10=$ very much enjoy. Likewise, patients were asked to recall the content of the VCD, one point was given to each content recalled. The maximum score was 5 .

\section{Data analysis}

Several statistical methods were used in data analysis. A paired $t$-test was carried out to determine whether significant difference existed between pain scores in relation to the use of visual stimuli. The effect of gender, age, location of residency, and the underlying medical conditions of the patients were compared by independent $t$-test and ANOVA respectively. Degree of enjoyment and memory of the content of VCD were correlated with the net improvement in pain scores using the Pearson correlation. A $p$ value $<0.05$ was considered statistically significant. The current study with 30 patients and a cross-over design has $80 \%$ power for an effect size of $0.3-0.4$ at $5 \%$ alpha. The effect size was calculated with reference to studies of Vessey et al. ${ }^{19}$ and Tse et al.,${ }^{20}$ with the effect size of 0.46 and 0.43 , respectively.

\section{RESULTS}

Thirty-three patients took part in the study (17 male, 16 female patients, age $75.8 \pm 9.8$ years). Of all patients, $33 \%$ (age $71.6 \pm 8.6$ years) were staying in

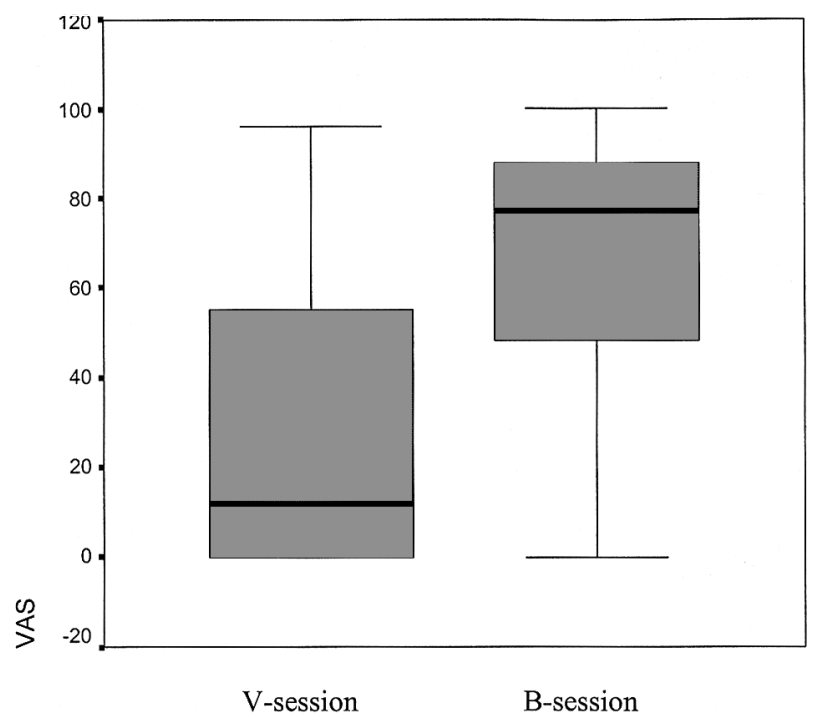

FIG. 3. A comparison of the mean perceived pain intensity between the $\mathrm{V}$-session and the B-session $(n=33)$.

their own homes and $67 \%$ (age $78.0 \pm 9.8$ years) were staying in nursing homes. All the participants were having leg ulcers and needed superficial debridement and wound dressing procedures.

There was a significant reduction in VAS during V-sessions (Fig. 3). The results $(t=-7.838$, d.f. $=32$; $p<0.001)$ indicated a more than twofold difference in pain scores, with the mean pain scores decreased from $67.7( \pm 29) \mathrm{sec}$ in B-sessions to $25.6( \pm 30) \mathrm{sec}$ in V-sessions. The net improvement in VAS was not found to be different between patients with different gender, residency or underlying medical condition (Table 1). In addition, a positive correlation was found between age and the net improvement in VAS $(r=0.379, p<0.05)$ (Fig. 4).

TAble 1. The EfFect of Gender, Location of Residency, AND UndERLYING MEDICAL CONDITION ON IMPROVEMENT IN VAS

\begin{tabular}{|c|c|c|}
\hline & Net improvement in pain scores, mean $( \pm S D)$ & $p$ value \\
\hline \multicolumn{3}{|l|}{ Gendera } \\
\hline Female $(n=16)$ & $43( \pm 33)$ & \\
\hline Male $(n=17)$ & $38( \pm 38)$ & 0.696 \\
\hline \multicolumn{3}{|l|}{ Location of residency ${ }^{a}$} \\
\hline Own home $(n=11)$ & $25( \pm 33)$ & \\
\hline Nursing home $(n=22)$ & $49( \pm 33)$ & 0.057 \\
\hline \multicolumn{3}{|c|}{ Underlying medical conditions $\mathrm{s}^{\mathrm{b}}$} \\
\hline $\operatorname{Nil}(n=11)$ & $54( \pm 31)$ & \\
\hline Old CVA $(n=15)$ & $33( \pm 35)$ & 0.271 \\
\hline $\mathrm{DM}(n=17)$ & $36( \pm 36)$ & \\
\hline
\end{tabular}

andependent samples t-test. A $p$ value of $<0.05$ was considered statistically significant.

bOne-way ANOVA was used. A $p$ value of $<0.05$ was considered statistically significant. 


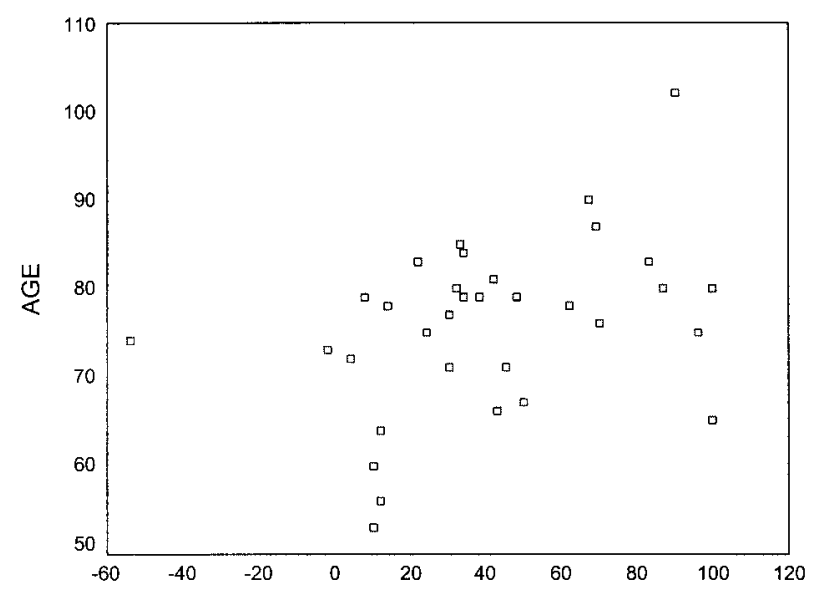

Net improvement in pain scores

FIG. 4. Net improvement and age.

The mean degree of enjoyment was 7.5 on a 10point scale, and the memory of the content of the VCD was 4.03 on a 5-point scale. There was no significant correlation of degree of enjoyment $(r=0.275$, $p>0.05)$ or memory of the content $(r=0.283, p>$ 0.05 ) of the VCD with the net improvement in VAS.

\section{DISCUSSION}

The study confirmed that visual stimuli generated by wearing an eyeglass display were a useful non-pharmacological means of pain relief in elderly patients for superficial debridement and wound dressing of leg ulcers. We have previously reported a $33 \%$ increase in pain threshold and $27 \%$ increase in pain tolerance while watching video via a television ${ }^{21}$ and a $52 \%$ increase in pain threshold and $40 \%$ in pain tolerance while watching video via an eyeglass display 20 in healthy volunteers with tourniquet pain study. In terms of the content of the visual stimuli, the majority of the patients chose to watch VCD of nature environment. Only four out of 33 patients chose to watch Chinese opera, and nobody chose watching cartoons. There would be insufficient data to elicit the effect of different content of visual stimuli on pain perception. Nevertheless, the results suggested the use of visual stimuli might be more effective when administered via the eyeglass display, which can block off unpleasant sights of the immediate environment, and create a pleasing environment by the video world.

The current study cannot elucidate the mechanism by which visual stimulation provides analgesic. However we postulate the effect observed may be related to the modification of the patient's visual input as well as distraction. It is suggested by Donald ${ }^{22}$ that nociceptive, exteroceptive and interoceptive sensory processes provide parallel contributions to pain. Unpleasant environmental factors, which constitute exteroception, produce anxiety and accelerate pain. ${ }^{23}$ Despite the fact that blank eyeglass also shields off unpleasant sight, it is suggested that pain relief may be more effective with visual input broadcasting via the eyeglass display, as the visual input may be served as a form of distraction.

The visual stimulation in this study may also provide analgesic by distraction. The exact biological actions and mechanisms underlying the effectiveness of distraction as a method of handling pain are generally unknown. ${ }^{24}$ In theory, a person's capacity for processing information is limited, and allocation of attention to one task limits the attention that may be given to another. ${ }^{25}$ McCaffrey and Pasero $^{26}$ propose distraction as "sensory shielding." The patient is shielded from the sensation of pain by increased sensory input from other sources. By exercising attention and concentration on stimuli other than pain, pain is placed on the periphery of awareness. As a result, when subjects with pain pay attention to the VCD via the eyeglass display, visual sensory input is increasing, and less attention is available for focusing on pain.

Distraction strategies are most appropriate for use over a brief period of time ranging from minutes to an hour, and for pain that is mild to moderate in intensity. ${ }^{26}$ Visual stimuli are predominant distraction strategy and especially useful for short procedures associated with intense anxiety and considerable pain. Examples include lumbar puncture, bone marrow aspiration, burn debridement, suture removal, painful intramuscular injection, difficult venipuncture, and closed reduction of a bone fracture. Visual stimuli may be a better option to analgesia than drugs in these conditions where the nociceptive stimulus is intense but brief, making drug titration difficult.

We also detected a positive correlation between age and the net improvement in VAS. Cognitive-behavioral therapy such as hypnosis, relaxation with guided imagery, distraction has been widely and successfully used with elderly patients in pain relief. ${ }^{27}$ Indeed, the most common pain-relieving cognitive strategies reported by the elderly patients were distraction technique. ${ }^{28}$

It is well known that elderly patients are more vulnerable to the adverse effects of analgesics such as sedation and respiratory depression. Our finding that the usefulness of visual stimulation increases with age makes it a particularly attractive 
analgesic option in this group of patients. In addition, opioid administration in the community may not be safe, as there are insufficient resuscitation facilities available. There is also an administrative inconvenience for the community nurse to carry dangerous drugs around for use in community patients during wound dressing. All these make the use of visual stimulation for analgesia-with its unique feature of simplicity, robustness, and safety and proved efficacy in elderly-very attractive in the present setting.

We found no difference in the improvement in VAS between male and female patients, and no difference between those living at home and those institutionalized. These suggest the current study can have a wide scope of application. The present study failed to demonstrate any correlation between the improvement in VAS and indicators for the patient's involvement in the visual stimulation such as degree of enjoyment or ability to recall contents of the video. It is possible analgesia by visual stimulation involves a mechanism other than distraction and so is unaffected by the degree of patient involvement. It is also possible that since most of our patients showed a high degree of involvement in the stimulation, and this relationship could not be evaluated effectively in the present study.

Further studies, specifically addressing physiological data to correlate with pain scores and visual stimuli, are needed. Also, the content of the visual stimuli could be more tailor-made such as putting pictures of the patients' loved ones, old family members, and friends, or places they had desired to visit into the VCD and project them via the eyeglass display. This might help patients to be more immersed in the video-stimulated world and become less aware of their pain. To sum up, with the application of visual stimuli via the eyeglass display, there was a significant decrease in pain perception during wound dressing and debridement for leg ulcers in elderly patients. Age was positively correlated with the net improvement in pain scores. Indeed, providing visual stimuli to patients requires no prescription by the physician, and is convenient to use and acceptable to patients, making the use of various visual stimuli an appealing non-pharmacological intervention for pain relief.

\section{ACKNOWLEDGMENTS}

We express thanks to the patients who participated in the study. We also thank Nelly Ho and all the nursing staff in the Community Nursing Unit of Kowloon
Hospital, Hong Kong, in helping and facilitating this study. And our gratitude goes to Professors Thomas Wong and Joseph Yang, who have given tremendous support and guidance in this study.

\section{REFERENCES}

1. Callam, M.J., Ruckley, C.V., Harper, D.R., et al. (1985). Chronic ulceration of the leg: extent of the problem and provision of care. British Medical Journal, 290:1855-1856.

2. Lindholm, C., Bjellerup, M., Christensen, O.B., et al. (1992). A demographic survey of leg and foot ulcer patients in a defined population. Acta DermatoVenereologica (Stockholm) 72:22-230.

3. Linholm, C., Bjellerup, M., Christensen, O.B., et al. (1993). Quality of life in chronic leg ulcer patients. Acta Dermato-Venereologica (Stockholm), 73:440-443.

4. Harahap, M. (1990). Differential diagnosis of leg ulcers. Clinics in Dermatology 8:1-3.

5. Dealey, C. (1999). The care of wounds: a guide for nurses, $2^{\text {nd }}$ ed. Oxford: Blackwell Science.

6. Gould, D. (1999). Wound management and pain control. Nursing Standard 14:47-54.

7. Walshe, C. (1995). Living with a venous leg ulcer: a descriptive study of patients' experiences. Journal of Advanced Nursing 22:1092-1100.

8. Ryan, I.J. (1985). Current management of leg ulcers. Drugs 30:461-468.

9. Holst, R.G., \& Krisofferson, A. (1998). Lidocaineprilocaine cream (EMLA Cream) as a topical anaesthetic for the cleansing of leg ulcers. The effect of length of application time. European Journal of Dermatology 8:245-247.

10. Casey, G. (1998). The management of pain in wound care. Nursing Standard 13:49-50, 53-54.

11. Melzack, R., \& Wall, P.D. (1965). Pain mechanisms: a new theory. Science 150:971-979.

12. Melzack, R. (1996). Gate Control Theory. On the evolution of pain concepts. Pain Forum 5:128-138.

13. Sparks, L. (2001). Taking the "ouch" out of injections for children: using distraction to decrease pain. American Journal of Maternal/Child Nursing 26:72-78.

14. McCaffery, M. (1990). Nursing approaches to nonpharmacological pain control. International Journal of Nursing Studies 27:1-5.

15. Melzack, R., Weisz, A.Z., \& Sprague, L.T. (1963). Stratagems for controlling pain: contributions of auditory stimulation and suggestion. Experimental Neurology 8:239-247.

16. Brown, C., Chen, A., \& Dworkin, S. (1989). Music in the control of human pain. Music Therapy 8:47-60.

17. Good, M. (1996). Effects of relaxation and music on postoperative pain: a review. Journal of Advanced Nursing 24:905-914.

18. Good, M., Stanton-Hicks, M., Grass, J.A., et al. (1999). Relief of postoperative pain with jaw relaxation, music and their combination. Pain 81:163-172. 
19. Vessey, J.A., Carlson, K., \& McGill, J. (1994). Use of distraction with children during an acute pain experience. Nursing Research 43:369-372.

20. Tse, M.Y.M., Ng, J.K.F., Chung, J.W.Y., et al. (2002). The effect of visual stimulation via the eyeglass display and the perception of pain. CyberPsychology $\mathcal{E}$ Behavior 5:65-75.

21. Tse, M.Y.M., Ng, J.K.F., Chung, J.W.Y., et al. (2002). The effect of visual stimuli on pain threshold and tolerance. Journal of Clinical Nursing 11:462-469.

22. Donald, P. (2000). Psychological and neural mechanisms of the affective dimension of pain. Science 288:1769-1772.

23. Moss, V. A. (1987). The effect of music on anxiety in the surgical patient. Perioperative Nursing Quarterly 3:9-16.

24. National Institutes of Health. (1996). Integration of behavioral and relaxation approaches into the treatment of chronic pain and insomnia. Journal of the American Medical Association 276:313-318.
25. Kahneman, D. (1973). Attention and fffort. Englewood Cliffs, NJ: Prentice-Hall.

26. McCaffrey, M., \& Pasero, C. (1999). Pain clinical manual 2nd ed. St. Louis, MO: Mosby.

27. Keefe, F.J., \& Williams, D.A. (1990). A comparison of coping strategies in chronic pain patients in different age groups. Journal of Gerontology Psychological Sciences 45:161-165.

28. Yates, P., Dewar, A., \& Fentiman, B. (1995). Pain: the views of elderly people living in long-term residential care settings. Journal of Advanced Nursing 21:667-674.

Address reprint requests to: Mimi M.Y. Tse, Ph.D. School of Nursing Hong Kong Polytechnic University Hung Hom, Kowloon, Hong Kong

E-mail: hsmtse@inet.polyu.edu.hk 